\title{
Designing a Fuzzy Inference System for Multi-Criteria Assessment of Urban Green Space Development (Case study: Shiraz Metropolitan Area)
}

\author{
Samaneh Shariati, Abdolhossein Zarifianmehr and Ali Amini \\ Think-tank expert at Shiraz Central Municipality, MA in Urban Planning, Faculty of Urban and \\ Architecture, Science and Research Branch, Islamic Azad University, Tehran-Iran. \\ *samaneh.shariati@yahoo.com
}

\begin{abstract}
Urban green spaces are recognized as key ecological service providers to urban dwellers with multiple functions and also an important pillar of sustainable development. The multi criteria nature and the uncertainty associated with the assessing of green space development make the issue as complex as cannot be addressed with conventional logical methods. One of the most important methods of multi criteria assessment is Fuzzy Inference System (FIS). This paper assesses urban green space development within Shiraz metropolitan area, Iran based on various criteria by designing a special fuzzy inference system. The data for the present study were collected through questionnaires and semi-structured interviews. The result revealed that increase in green space area and the per capita allocated to each person and subsequently the improvement in their distribution pattern in the last decade have changed the status of the majority of regions' green spaces toward relatively developed and definitely developed regions. Predictions and strategic analysis of the developing green space found that, if the growth rate of green space area exceeds $30 \%$, region 7 will be a relatively developed region and if the increase in the area of this land use exceeds $45 \%$, it will be definitely a developed region.
\end{abstract}

Keywords: Green Spaces, Multi criteria, Fuzzy Inference System

\section{INTRODUCTION}

Urban green space includes everything in cities that has the vegetation. Collectively it is sometimes referred to as 'Green Infrastructure', encompassing the entire working landscape in cities that serve roles such as improving air quality, flood protection and pollution control (Girling and Kellett, 2005). Urban green spaces are also of a strategic importance for improving the quality of life of our increasingly urbanized society and increasing sustainability in cities. Since more people are chosen to live in the city, the pressure on current green spaces is rising. The author Jane Jacobs (1967) claims that green spaces, in particular parks, are the most valuable, as they often stand in direct contrast to a very compact and busy city life. She argues that green areas provide the crowded city with certain functions, and agree with modernist planners who considered parks as "self-evident virtue" (Jacobs, 1961). Therefore, Green areas must be planned along with other city policies because they are important spaces that maintain the quality of the urban environment. Since urban green space is associated with multi-dimensional values, the quality of its development requires a careful empirical assessment based on different criteria. Beside this, subjects such as development and sustainability have never been so discussed and need a comprehensive framework when assessing their quality relating to green space, because the imprecision and uncertainty appear to be a general characteristic of development. There are several conventional methods such as density function, index analysis, and multivariate analysis which were applied in previous studies in order to development analysis and assessment. However, the uncertainty associated with the identity of development issues leads to the inefficiency these methods (McDonald, 1987 and Feng et al., 1994). In fact, the uncertainty and the multi criteria nature of development is known as fuzzy uncertainty. 
According to Klir and Folger Fuzzy (1988) uncertainty relates to the events that have no well-defined, unambiguous meaning and multicriteria nature. Fuzzy Set Theory (FST), a powerful tool used to cope with this complexity, imprecision and uncertainty, can be used to handle linguistic concepts, human feeling and perform nonlinear mapping between inputs and outputs. Therefore the benefits of using fuzzy set theory brings with it the attention features and the ability to deal with linguistic terms which explains its adoption in multi criteria assessment of green space development.

Moreover, fuzzy set theory is widely accepted for capturing expert knowledge. It allows us to describe the expertise in more intuitive, more human like manner and without thinking in terms of mathematical models. In this regard, Fuzzy Inference Systems (FIS) are one of the most famous application of fuzzy set theory and can cope with all the above-mentioned problems (Teodorovic, 1999 and Bonivento et al. 1998). The fuzzy inference system can also circumvent the deficiencies of existing conventional methods. Generally, the advantage of applying FIS for evaluating the degree of development is because of the following mechanism (Lin, 2007): The fuzzy inference system can circumvent the deficiencies of existing methods for analyzing activity centers via the following mechanisms: First, the premise of the fuzzy rule can use multiple criteria. Second, the rule base and membership function are both established via the questionnaire survey of individual judgments, which can be used to establish the goodness of fit between the model judgments and individual judgments. Third, the linguistic variables, other than statistical variables, used in fuzzy rules make the inference process more closely approach the judgment process of individuals. Finally, the linguistic outputs are useful for clearly describing and discussing the decision making process.

Therefore, in this study, a comprehensive fuzzy inference system is designed to assess the degree of green space development based on multiple criteria in 2001 to 2011 and then to forecast this degree in 2011 to 2022 in order to restructure the development processes of new green spaces, and make optimal decisions on the quality of development in future. To achieve the research objectives concerning the evaluation and projection of the development of green space development, research data were collected through field collection practices, questionnaire and interviews. In this study, just one questionnaire was used for generating fuzzy rules by surveying experts familiar with the range of regions under study. A total of 50 experts comprise the sample size in this questionnaire. The present study uses cluster sampling and a semi-structured interview based on which the survey continues with each of the experts to achieve theoretical saturation (Strauss and Corbin, 1990).

\section{LITERATURE REVIEW}

Over the past three decades research on urban green spaces, in particular parks, has flourished. There is now a considerable body of scholarly work on urban green space, covering topics such as design, use of green space, green space evaluation, green space values, environmental equity and the like. In this review, we limit our discussion to topics that are relevant to urban green space and development topical area such as assessing, locating, etc. A summary of the various applied tools (such as fuzzy set theory) in different research may benefit researchers in the urban and green space field.

In one case, Rezaeiniya et al. (2014) used fuzzy analytic network process (FANP) for greenhouse locating in Iran. The research considered several factors for greenhouse locating, like: heating supply, greenhouse expansion plans, access to different facilities, land cost, access to proper transportation, proximity, etc. They used the Fuzzy Extent Analysis for capturing the human's appraisal of ambiguity, tolerating the vagueness of problem and making the appropriate decision (Rezaeiniya et al. 2014).

The other work by Zhan et al. (2000) integrated GIS and Fuzzy Set Theory to classify urban land uses especially urban green spaces. They used fuzzy memberships and fuzzy rules to deal with uncertainty. They also stated that the final result of such a classification process would consist of a land use map and a corresponding 
map indicating uncertainty levels of the assignment of area to the relevant classes. The proposed approach would be based on estimating the real cover of an area by features like green space, water body, built up area etc. From these cover compositions the major functions would be inferred in a land use map (Zhan et al., 2000).

$\mathrm{Hu}$ and Wang (1998) developed an AHP fuzzy evaluation technique for "integrated ecological service functions" (IESF) for green space. In fact, putting forward on IESF, they analyzed and evaluated social, economic and ecological dimensions of urban green spaces. They implied that such combination of fuzzy set theory and AHP may help scientifically manage and plan the pattern of urban green space and promote the building of sustainable cities ( $\mathrm{Hu}$ and Wang, 1998).

Finally, the study by Givi et al. (2015) applied a fuzzy AHP method for park site selection in Tehran metropolitan area, Iran. Their ultimate goal of this research was to identify suitable locations for the construction of parks based on the FAHP model to help planners and decisionmakers in the region 7 of Tehran municipality. For this they extracted required criteria, then compared those using FAHP and finally prepared Fuzzy maps overlaid in GIS to identify suitable locations (Givi et al., 2015). Focusing on all the related studies, shows while there have been many applications of fuzzy sets theory in the green space field, only limited number of cases
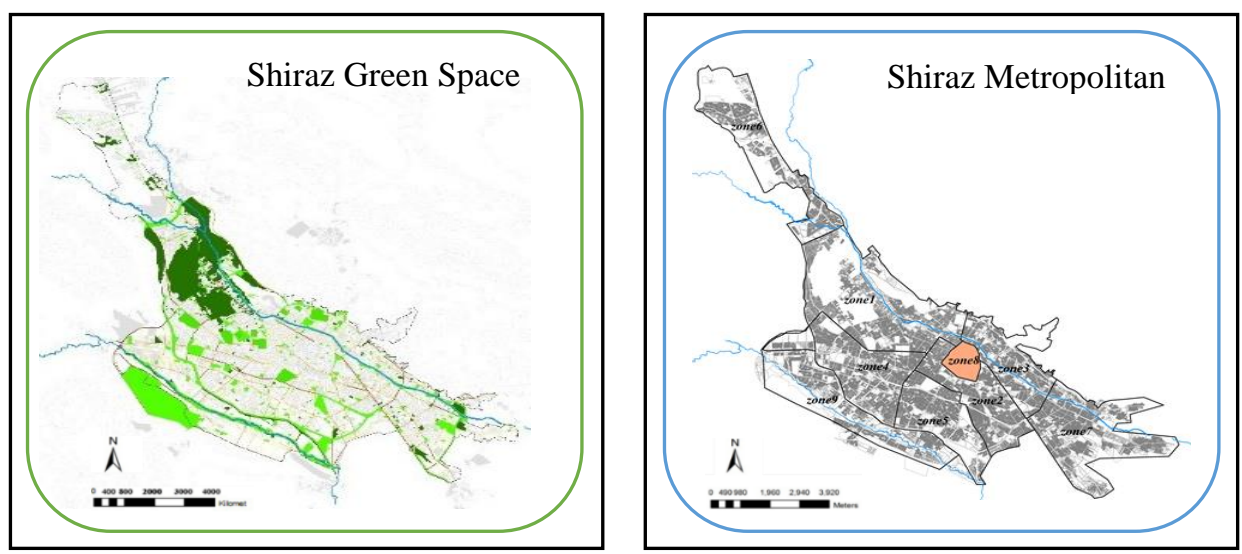

Figure 1: Left: Shiraz Green Space, Right: Shiraz Metropolitan Area can be found about including fuzzy inference system as well. This research attempt to address this gap in the literature by assessing the green space development using fuzzy inference system.

\section{CASE STUDY}

Shiraz with an area of 193 square kilometers and a population of over 1.4 million people, is the fifth populous city of Iran and is the capital of Fars province in south of the country. The metropolitan area of Shiraz has relatively high growth in terms of population and territorial expansion. This phenomenon is associated with increased needs for qualified green spaces which can lead to sustainable development, environmental promotion and improved satisfaction of local resident. The study area covers the eight regions which many of them have experienced increased residential densities in recent years placing pressure on parks. Urban development plans of most metropolitan of Iran reveal some forms of typology that has been applied to classify parks upon the size of park and the catchment area (Fig. 1). This typology can variously described as urban parks, regional parks, district parks and neighborhood parks. Generally, Shiraz city comprise 2429 hectare public green spaces which about 285 hectare is allocated to the all kinds of parks. This significant area allocated to parks puts a basic necessity on the careful assessment of their qualification. 
The study approach is illustrated in Fig. 2 and comprises four major parts: Evaluation criteria for green space development, membership functions for the linguistic variables, fuzzy rules, and fuzzy inference system.

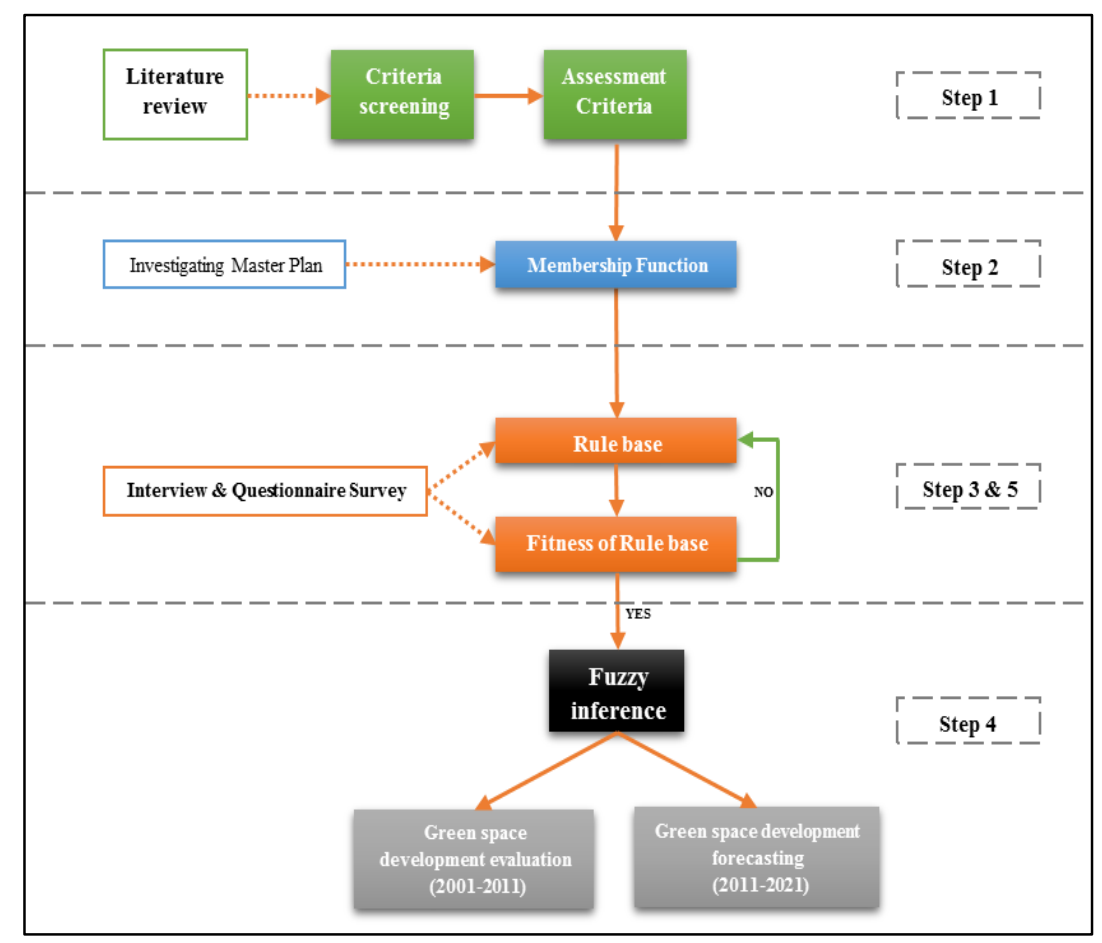

Figure 2: Assessment Approach

\section{Step 1. Determination Assessment Criteria}

As Alberti and Mega have stated (Alberti, ..., and Mega, ...), the criteria like accessibility of green space, per capita, and the percentage of region area allocated to green space (occupancy area) are the most important quantitative indicators of urban green space toward urban sustainable development. Therefore with reference to the use of fuzzy inference system for green space multiple criteria evaluation in different regions, the very important criteria are accessibility of green space, per capita, and the percentage of region area allocated to green space (occupancy area). Therefore, from the view point of all the above criteria and their characteristics, the region with high rates of accessibility, per capita, or occupancy area related to green space is likely to be a developed region with regard to green space. Based on the considerations of effective description, interaction with green space characteristics and data accessibility three above-mentioned criteria, are applied to evaluating the development levels of green space for each region.

In order to generating fuzzy values, each criterion in normalized using the following function (Shariati, 2012):

$$
x=\frac{B_{i j}-\min }{\max -\min }
$$

Where $\mathrm{i}$ represents the region under study, $\mathrm{j}$ represents evaluation criteria, and $\mathrm{Bij}$ represents the value of each criterion in each region.

\section{Step 2. Definition of Membership Function}

Membership values can be expressed in different types of membership function. Suppose $X$ is a universal set, for each fuzzy set like A, the membership function $\mu_{\mathrm{A}}$ is defined as following (Dubois et al, 2000):

$$
\begin{gathered}
A=\left\{x, \mu_{\mathrm{A}}(\mathrm{X}) \mid x \in X\right\} \\
\mu_{\mathrm{A}}(\mathrm{x}): X \rightarrow\left[\begin{array}{ll}
0 & 1
\end{array}\right]
\end{gathered}
$$


Here, the value of $\mu$ indicates membership value or degree $(x \in X)$ and membership value indicates the degree of membership of $x$ to the fuzzy set A or the linguistic degree $\mathrm{x}$ (Tanaka, 1962). First of all in fuzzification, we define the linguistic variables and determine the fuzzy range. The linguistic variables in this study are already determined with regard to available data in terms of the characteristics of urban green space. However, since the purpose of fuzzy control is to simulate a knowledge base, a group of experts was asked to choose certain linguistic degrees from among study variables according to approved plans and personal knowledge. In this way, three linguistic degrees (low, medium, and high) were specified for these linguistic variables or criteria. After defining the linguistic degrees of each set, membership functions were defined. These functions are sequential and continuous considering the nature of linguistic variables and they determine the degree of membership of each point in the set. For fuzzy variables, we can select one of the types of membership functions uniform, triangular, trapezoid or arch. Considering the nature of this study, the form of the functions is triangular. Thus, according to this discussion and Pedryce's theories (Pedryce, 2008, 329-325), the knowledge and experience of experts are the basis for the formation of membership functions. After feeding the values of linguistic variables (fuzzy amplitudes) into the MATLAB software (The Math works Inc., Natick, Mass.), membership functions were constructed.
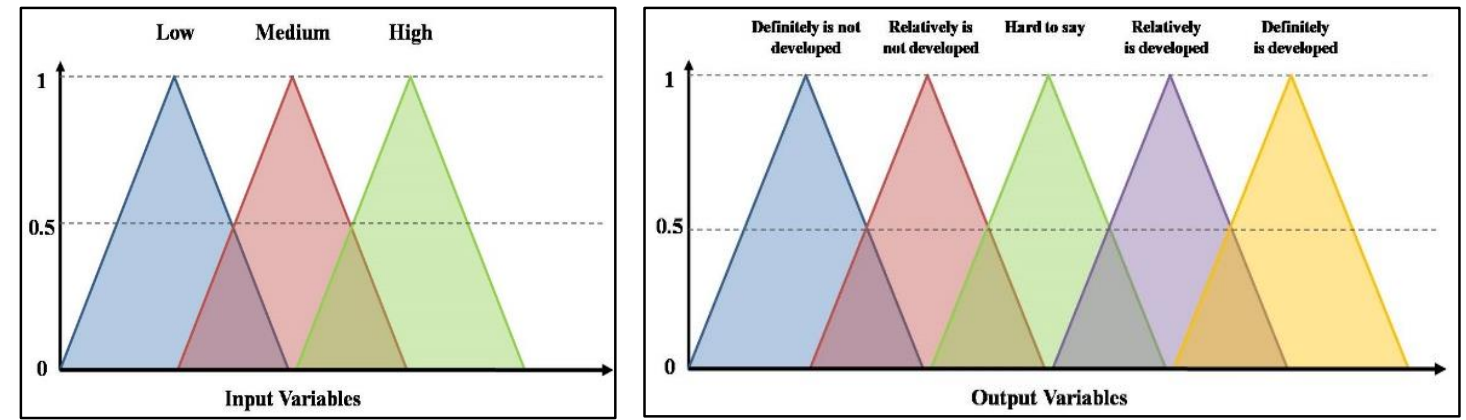

Figure 3: Left: Membership of Input linguistic, Right: Membership of Output

\section{Step 3. Generation of Fuzzy Rules}

The application of fuzzy logic in real world system is mainly used with fuzzy IF-THEN linguistic rules, which describe the logical evolution of the system based on the linguistic variables (Leung, 1988). In fact, IF-THEN fuzzy rules are conditional statements that show the association of one or more linguistic variables to each another. One simple rule is shown as following (Dubois et al, 1996): IF < premise> THEN<consequence>

Whereby both premise and consequence are characterized by fuzzy or linguistic elements respectively.

For example for the fuzzy rule: IF the green space per capita is high, THEN the region is definitely developed, the phrase between IF and THEN is the premise (or antecedent), and the phrase after THEN is the consequence (or conclusion) (Tanaka, 1962). In this study fuzzy rules were generated based on literature review, approved plans, and a questionnaire survey of 50 professional workers (experts) in Shiraz. Workers responses for each activity center were graded according to five linguistic degrees: "Definitely is developed", "Relatively is developed", "Hard to say", "Relatively is not developed", and "Definitely is not developed". Finally, the identification and determination of the levels of green space development based on the questionnaire surveys and the linguistic performance criteria were combined to generate twenty seven fuzzy rules, as a short list of it is shown in Table 1.

Table 1 lists the rule base for identifying the levels of green space development and presents the following findings: First, the criterion of green space per capita dominates the determination of the levels of development and 
presents few trade-off relationships with the other two criteria. It can be concluded that green space per capita is very important for the determination of the levels of development. Second, significant trade-off relationships exist between accessibility and percentage of region area allocated to green space, meaning these two criteria are interdependent for the purpose. Finally, the progression relationships between premises and conclusions are discrete and irregular.

Table 1: Rule Base for the assessment of green space development

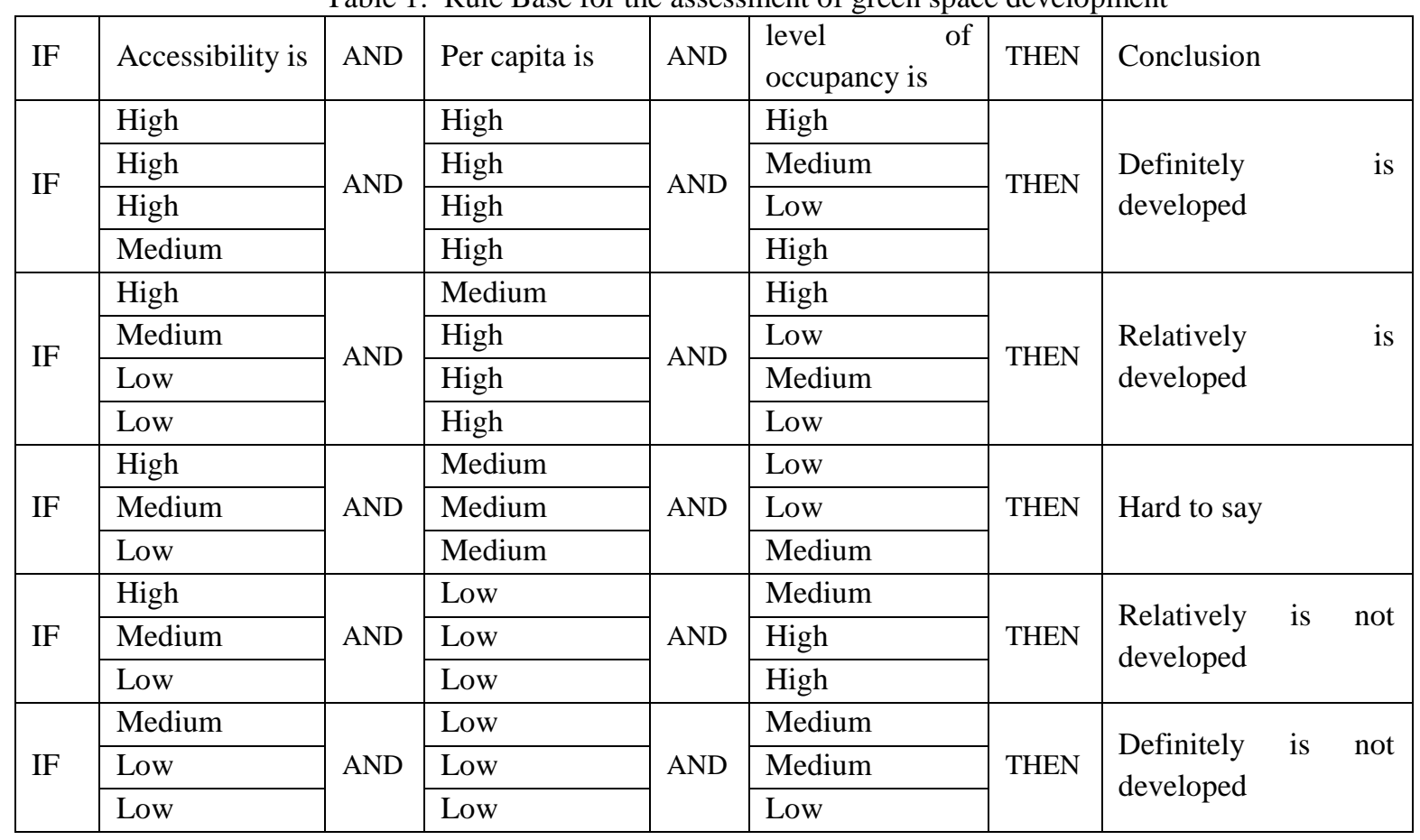

The following examples represent some membership functions which are used in fuzzy inference to assess the development of green space.

$$
\begin{aligned}
& \mu \text { definielyis developed }=\min \left\{\mu_{\text {high }}\left(x_{1}\right), \mu_{\text {high }}\left(x_{2}\right), \mu_{\text {high }}\left(x_{3}\right)\right\} \\
& \mu \text { relatively is developed }=\min \left\{\mu_{\text {high }}\left(x_{1}\right), \mu_{\text {medium }}\left(x_{2}\right), \mu_{\text {medium }}\left(x_{3}\right)\right\} \\
& \mu \text { Hard to a say }=\min \left\{\mu_{\text {high }}\left(x_{1}\right), \mu_{\text {medium }}\left(x_{2}\right), \mu_{\text {low }}\left(x_{3}\right)\right\}
\end{aligned}
$$$$
\mu \text { relatively is nat developed }=\min \left\{\mu_{\text {high }}\left(x_{1}\right), \mu_{\text {low }}\left(x_{2}\right), \mu_{\text {high }}\left(x_{3}\right)\right\}
$$$$
\mu \text { definitely is nat developed }=\min \left\{\mu_{\text {medium }}\left(x_{1}\right), \mu_{\text {low }}\left(x_{2}\right), \mu_{\text {low }}\left(x_{3}\right)\right\}
$$

\section{Step 4. Conduct of Fuzzy Inference}

Fuzzy inference system is essentially knowledgebased system that utilizes all the concepts which have been described in the previous sections: fuzzy logic, fuzzy IF-THEN and membership functions (Lenug, 1988). Based on the fuzzy rules, this study applied the Mamdani fuzzy inference system (Mamdani and Assilian 1975) to conduct fuzzy inference. The main component of Mamdani FIC are as following (Abraham, 2005):
1) Knowledge base: knowledge base stores all data, information, rules and relationships which are used by expert system, and one of the methods for representing knowledge in knowledge base is using If-Then rules. By combining these rules, it is possible to solve complicated problems.

2) Fuzzification interface: fuzzification inference receive the certain inputs and define how related they are to 
appropriate fuzzy sets in dependency rules.

3) Defuzzification interface: the input of a defuzzifier is a fuzzy set, and the output is a certain amount. In fact, in this section, the rules that are consistent and compatible with the degree of membership of each function become activated and the output of the inference engine, which is a membership function, is turned into a defuzzified value (Zahedi, 1999). Defuzzification is carried out in different ways such as the center of gravity method, the center of aggregates method, the height method, the center of the largest surface method and the average maximum method. The method used in this study is the center of gravity method (COG).

4) The inference engine: inference system is in fact the brain of the expert system which processes the stored rules and knowledge. The inference engine can be established based on different logic like fuzzy logic and it usually employ statistical computations for fulfilling its tasks.

The process and output of the fuzzy inference model used in this study are presented in figure 4. The emerging concepts and issues will be discussed in the Appendix.
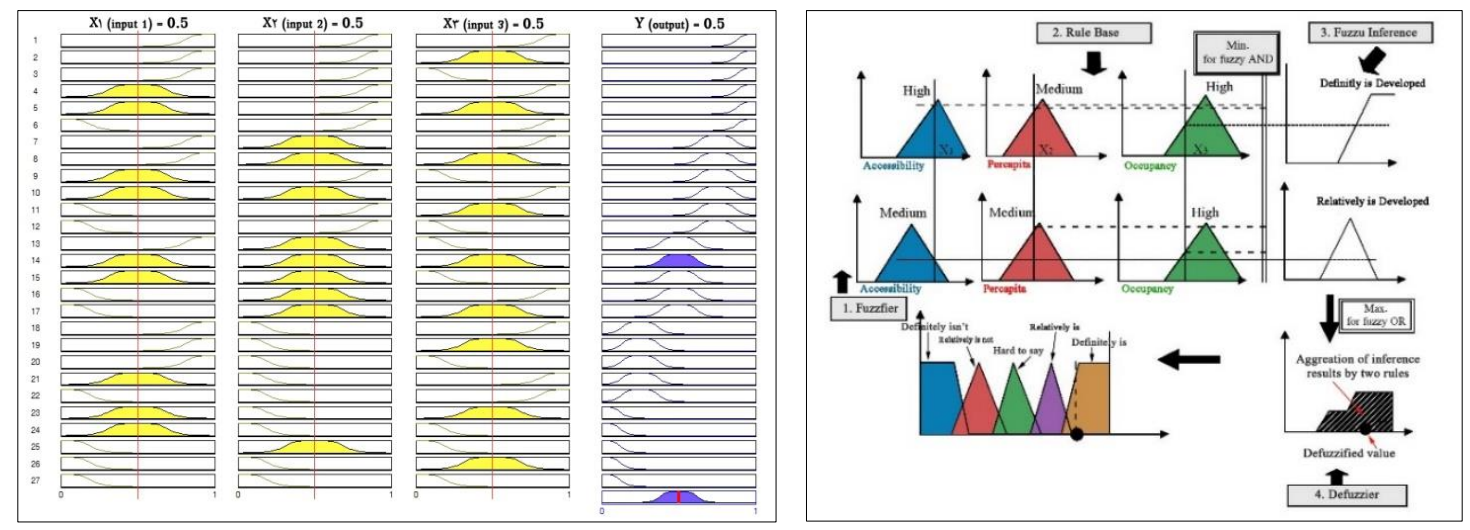

Figure 4: Left: Inputs (Xi) and output (Y) of the fuzzy inference, Right: Mamdani fuzzy inference system illustrated by the inference on green space development

\section{Step 5. Conduct of Fitness Test}

The fitness between the inference conclusions determined by the fuzzy inference system and the real recognition identified via the questionnaire survey should be examined to assess the validity of the study method (Mamdani and Assilian, 1975). The fitness is measured as follows:

$$
\text { Fitness }=N s / N
$$

Where $\mathrm{N}=$ total number of assessed region and Ns=number of regions for which the inference conclusions and survey results are the same. The fitness values range between 0 and 1 , and the validity of the inference system increases with the fitness value. The fitness of the inference for green space development is 0.62 in 2001, and about 0.78 in 2011, which represents an acceptable level.

\section{DISCUSSION}

The present study investigates the development of public green space in urban areas, separately. To this end, the development of these green spaces (parks) during 2001-2011is analyzed and predicted for 2021. Figure (5) shows changes in the development of public green space or parks during the period of 2001-2011. As can be seen in the comparative diagram of fuzzy inference, by increasing the areas allocated to parks and thereby increasing access of a region's residents to parks and also increasing per capita during the period of 2001-2011, regions 2, 3, 4, 5, 6 and 7 each have achieved a level of green space development. It is important to note that green space of all regions has achieved different levels 
of development. For example, the green space of region 5, which was nearly undeveloped in 2001, is now nearly developed by proper planning and allocating more areas for green space. Despite the uncertainty about the development or non- development of green space of region 6 in 2001, it has now achieved a level of development that can be classified as one of the developed regions in terms of green space in 2011.

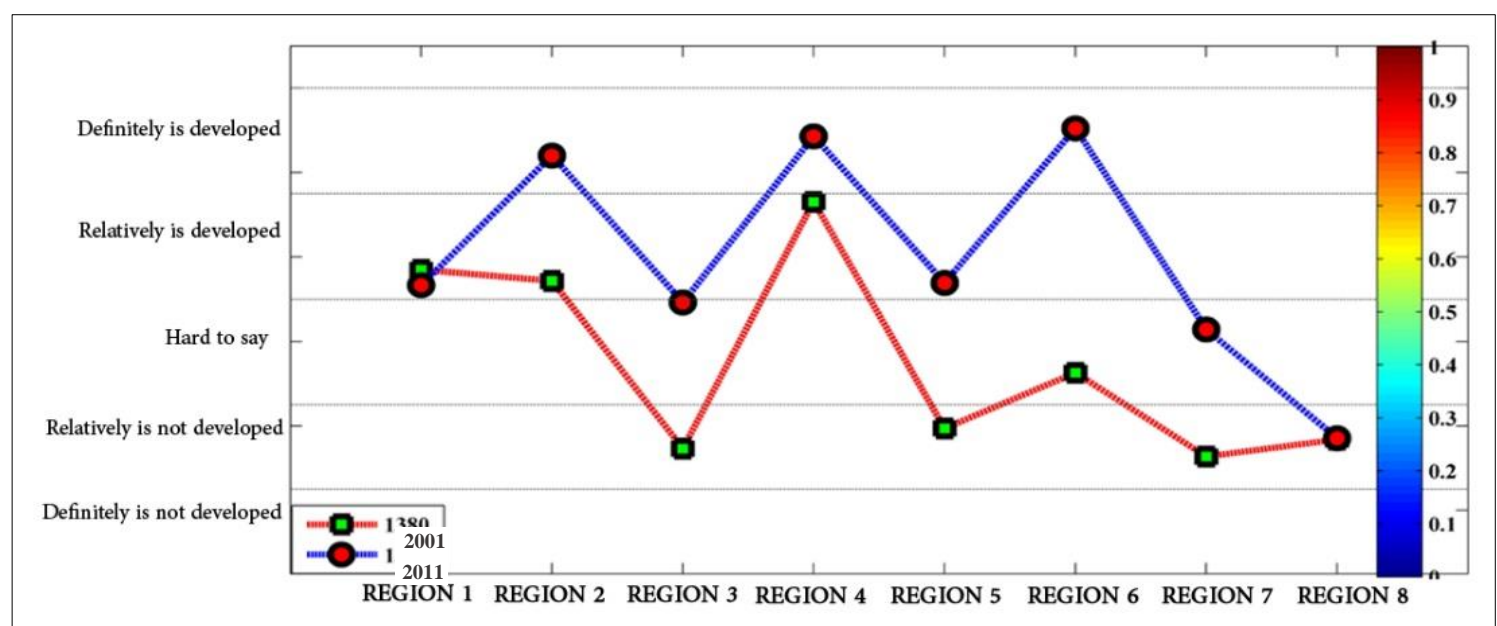

Figure 5: Fuzzy inference result for the assessment of green space development in 2001 to 2011

With the gradual increase in population during 2001-2011 and not increasing areas of public green spaces in proportion to the population, region 1 which is one the central regions of Shiraz city and has the highest rate of private and passive green space, is now in an unknown level of development and is now a place lower than 2001 with a relatively declining process. Unlike other regions, there has been no change in the status of green space in the past decade in the cultural-historical region (region 8 ). It is worth noting that the rate of obtained accessibility to the total parks of region 8 is in a relatively better situation than parks of other regions in the city. However, the other quantitative indicators of this region such as per capita of park and share of green spaces of the region total area are poorer compared to other regions.

\section{FORECAST AND STRATEGY}

Since the measured criteria have quantitative character, it would be better to use quantitative methods (like FIS) to cope with quantitative prediction and then make decision on policy directions. In fact, by taking advantage of FIS capabilities the research can reach quantitative policies like; by how much development in quantitative criteria or indicators, the regions of Shiraz can reach a very good level of development?

As already mentioned, some regions of Shiraz city are becoming developed areas regarding this specific land use with the development of quantitative indicators of public green space. If any of these indicators continuously increase and grow to a certain level in the coming years, these regions will definitely reach a very good level of development. Since today, green space is significantly important in improving the environmental situation and plays an important role in the creation of beautiful and pleasant scenery, reduction of pollution and protection of natural environment and its resources, proper assessment of the current qualitative and quantitative conditions and predicting and simulating the future is one of the important requirements of conscious management of this important urban land use. As was previously mentioned, levels of development can be analyzed and assessed based on three criteria of: accessibility, per capita and the occupancy area. According to the study conducted by Lin (Lin et al., 2006), since the increase in the urban population as well as the increase in the area of green space change the 
other two criteria (per capita and accessibility), this study, like similar experiences in this field, considers an acceptable approach to take account of the quantitative increase of the mentioned criteria.

This approach assumes that the area of green space (parks) will increase and change in the decade from 2011 to 2021 similar to the percent of changes from 2001 to 2011 (i.e. $75 \%$ ). The rate of change can be classified into five periods over a period of ten years. Therefore, it is considered that in each period of 2 years, the area of green space in each region will increase by $15 \%$ and subsequently, $15 \%$ of the ten-year growth rate of the population indicator will be realized in each two consecutive years. Then, the per capita and accessibility will increase to a certain level according to the calculations conducted for two indicators of area and population and finally, the level of the green space development will be predicted for 2021 for each region separately. Figure (6) shows the results of fuzzy inference for different growth rates of the desired criteria in the three years of 2001, 2011 and 2021.

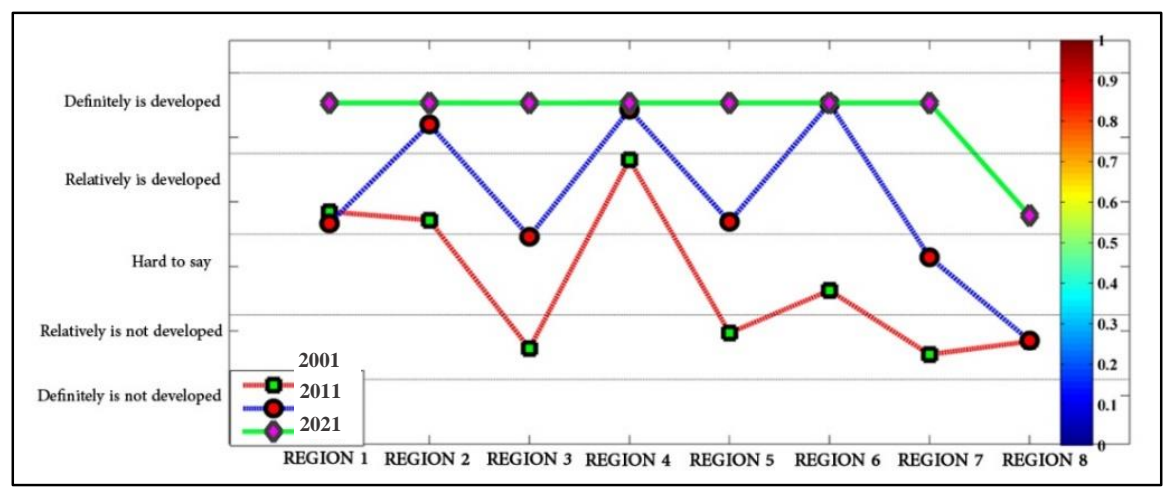

Figure 6: Fuzzy inference result for the assessment of green space development in 2001, 2011 and 2021

In addition to the overall forecast of the status of regions' green space in the horizon of years, the development of green space over the next ten years for the different growth rates of criteria is reviewed and drawn in Matlab. Figures 7 to 9 show the process of forecasting for developing green space of regions 1, 3, 5, 7 and 8. For example, according to figure 7 , if the growth rate of green space area exceeds $30 \%$, region 7 will be a relatively developed region and if the increase in the area of this land use exceeds $45 \%$, it will be definitely a developed region. In fact, assuming that the rate of increase in the mentioned criteria is observed and population increase is taken into account, this region will be recognized as a developed region in terms of green space during 2017-2021.

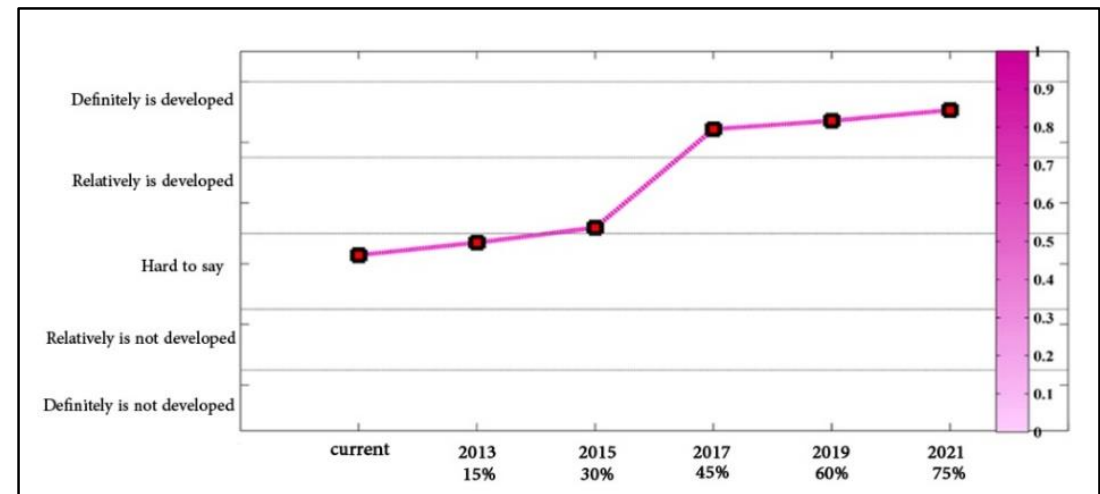

Figure 7: Forecasting result for the development of green space for region 7(2011-2021) green space development of regions of Shiraz, in

In general, according to the results of fuzzy inference, urban planners are suggested to consider plans to accelerate interventions and particular region 8 , and then to re-study and reinvestigate related regulations and opportunities in these areas and also increase the 
area of public green space in order to achieve sustainable development goals. Also, beside the quantitative development of green space in these regions, they can properly increase and provide environmental qualities that are consistent with the needs of residents of a region and are in line with the principles based on sustainable development.
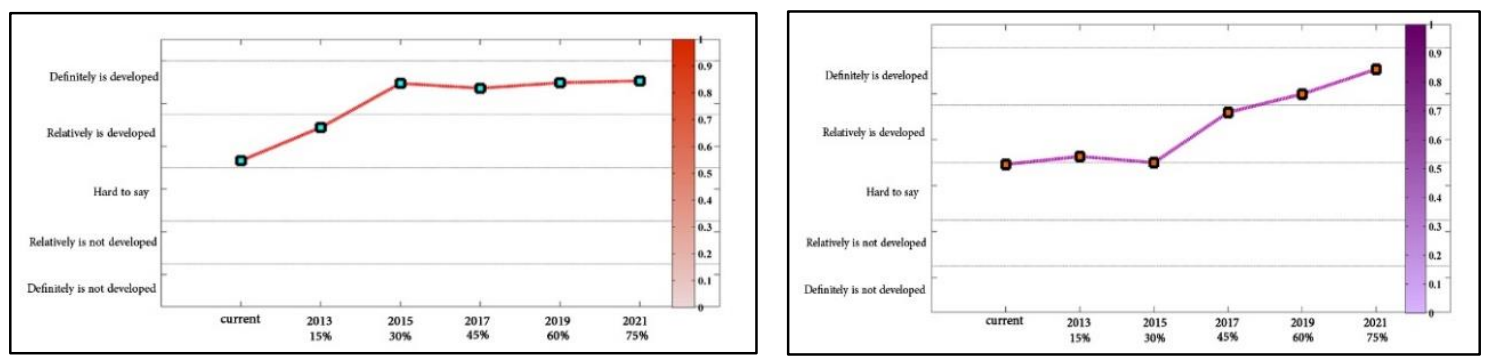

Figure 8: Forecasting result for the development of green space (2011-2021); left: region 1 and right: region 3
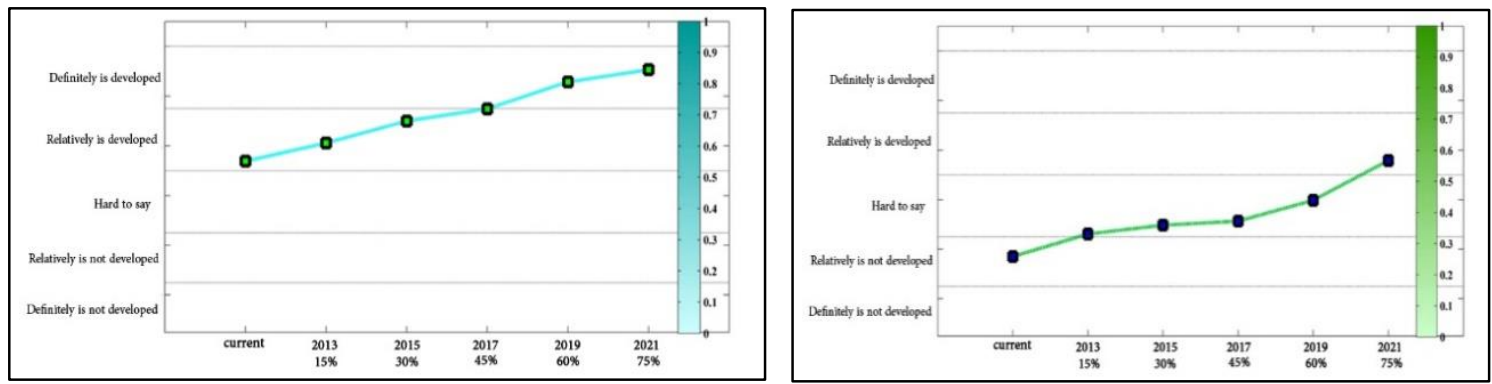

Figure 9: Forecasting result for the development of green space (2011-2021); left: region 5 and right: region 8

\section{CONCLUSION}

Shiraz metropolitan area has nine regions which are located in different areas with different levels of development. Some regions of the city such as region 8 are located in areas (central regions) that were physically fully developed in the past and have development constraints due to environmental and physical conditions. And some regions -such as regions 3,6 and 7- of the city are located in areas that are not fully developed and have the ability to accept new functions such as active and desirable green spaces under the implementation of urban development programs and projects. In general, research findings show that increase in green space area and subsequently, the per capita allocated to each person in a decade has created desirability for regions in terms of having green space. For example, increasing the quantitative indicators of occupancy level and per capita of parks in these areas has improved the accessibility of the residents. Improvement of the level of utility is manifested in the form of increase in the number of visitors of parks, increase in ecological and environmental qualities, and improvement of visual effects. Finally, the conscious planning for green space growth indicators and improving their distribution pattern in the last decade has changed the status of the majority of regions toward relatively developed and definitely developed regions. It is noteworthy that most of the changes are in accordance with the ratified development projects and plans.

In addition to the above results, development of green space of regions in the coming years can be determined and stimulated using the fuzzy inference system. For example, according to the results of inference, region 7 of Shiraz city may achieve levels of green space 
development that can be considered among the definitely developed regions. From the view point of sustainable development principles and due to the nature of public green spaces, i.e. parks, green space development in this region and other similar regions that contain a large part of urban residents are useful in the creation of the most efficient spaces for leisure time and increased social interactions, ecological balance in urban environment and ultimately achieving levels of sustainable development.

It is also noted that the use of fuzzy inference systems to conduct the intended analysis of the study has three major advantages compared with the conventional methods: first, the linguistic variables used in the system can indicate the ambiguity and uncertainty of personal feeling. Second, fuzzy rules and membership functions obtained from the questionnaires and interviews, can well avoid subjective bias of the researcher. And third, providing the inference results in the form of linguistic variables makes it easy for the public understanding and subsequent analysis and applications.

Since the compatibility of fuzzy rules is acceptable and intended in this study, the validity of fuzzy rules can be increased in further studies using the following ways: 1) increase sample surveys to create a comprehensive rule base.2) In addition to producing more comprehensive rules, use of more criteria and linguistic degrees can also describe the real world more accurately.3) Advanced and systematic methods, like neural networks and genetic algorithms, can be used to develop fuzzy rules to increase the accuracy and efficiency of the analysis process.

\section{REFERENCES}

Abraham, A. (2005). Adaptation of Fuzzy Inference System Using Neural Learning, Stud Fuzz 181, 53-83.

Alberti, M. 1996. Measuring Urban Sustainability, Journal of Environ Impact Assess REV, Vol. 16: 381-424.
Bonivento, C., Fantuzzi, C., and Rovatti, R., eds. (1998). Fuzzy logic control: Advances in methodology, World Scientific, London.

Dubois, D., and Prade, H. (1996). What are fuzz rules and how to use them? Fuzzy Sets and Systems, 84(2), 169-185.

Feng, C. M., Chen, J. C., and Hwang, T. H. (1994). "The population density functions of Taipei metropolis." Paper presented at the 5th Annual Conf. of Chinese Regional Science, Taipei, Taiwan.

Girling C, Kellet R. Skinny Streets and Green Neighborhoods: Design for Environment and Community. Washington, Island Press. 2005

Givi A. A, Karimi S, Foroughi N, Moarab Y, Nikzad V. Using Fuzzy Logic Analysis in GIS and FAHP Method for Parks Site Selection in Urban Environment (Case Study: Region 7, Tehran Municipality). Curr World Environ 2015; 10(2). Available from: http://www.cwejournal.org/?p=11251

Hu. D., and Wang. R. (1998). An integrated approach to evaluate on ecological service functions for urban green space and its application. Journal of Environmental Science. Vol. 10, No. 3, pp. 316324.International Inc., New Jersey.

Jacobs, J., 1961. The Death and Life of Great American Cities. Pimlico, London.

Klir, G.J., Folger, T.A., 1988. Fuzzy Sets, Uncertainty, and Information. Prentice-Hall

Leung, W. (1988). Fuzzy consepts in expert systems. Computer, Vol. 21, pp.43-56.

Lin. J. J., Feng, C. M. and Hu. Y. Y, (2006), Shifts in Activity Centers along the Corridor of the Blue Subway Line in Taipei, Journal Of Urban Planning And Development, Vol. 132, No. 1, PP 21-28.

Mamdani, E. H., and Assilian, S. (1975). “An experiment in linguistic synthesis with a fuzzy logic controller.” Int. J. Man-Mach. Stud., 7(1), 1-13.

McDonald, J. F. (1987). "The identification of urban employment sub center." J. Urban Econ., 21(2), 242-258.

Mega, V., Pedersen, J., 1998. Urban sustainability indicators, European Foundation for the Improvement of Living and Working Conditions. 
Pedrycz, W. (2001), "Fuzzy equalization in the construction of fuzzy sets". Fuzzy Sets and Systems. Vol. 119, pp. 329- 335.

Rezaeiniya, N., et al., 2011. Fuzzy ANP approach for new application: Greenhouse location; a case in Iran. Journal of Mathematics and Computer Science, Vol. 8, 1-20.

Shariati, S. (2012). Exploring the Spatial Impacts of Tehran Subway System, M.S Thesis in Urban and Regional Planning, supervised by Dr. A. Soltani. Shiraz University.

Strauss, A. and Corbin, J. (1990), Basics of Qualitative Research: Grounded Theory Procedures and Techniques (2nd Ed.). Newburyo Park: SAGE Publications.
Tanaka, C. (1962), Fuzzy Set Theory \& its Practical Applications, translated by Ali Vahidian Kamyad and Hamed Reza Tareghian, Ferdowsi University Mashhad, publication No.327.

Teodorovic, D. (1999). "Fuzzy logic systems for transportation engineering: The state of the art." Transp. Res., Part A: Policy Pract, 33(3), 337-364.

Zahedy, M. (1999). Fuzzy Set Theory and its Applications, Tehran, University Publication.

Zhan. Q., Molenaar. M., and Gorte. B. (2000). International Archives of Photogrammetry and Remote Sensing. Vol. XXXIII, Part B7. Amsterdam 